\title{
P-0751- Prediabetes Rate in Eastern Anatolia, Turkey: The rate of Diabetic Retinopathy
}

\author{
Doğan Nasır Binici', Özge Timur ${ }^{1}$, Pınar Tosun Taşar², Murat Filiz¹, İdris Baydar \\ ${ }^{1}$ Region Training and Research Hospital, Department of Internal Medicine, Erzurum, TURKEY
}

${ }^{2}$ Region Training and Research Hospital, Department of Geriatrics, Erzurum, TURKEY

\section{INTRODUCTION:}

Diabetic retinopathy (DR) is the most important cause of blindness in adult diabetic patients. DR is a spesific vascular complication of both types of diabetes. The prevalence of DR is related to duration of diabetes and glycemic control. Chronic hyperglicemia, nephropathy, hypertension and dyslipidemia increases the risk of DR.

The preventive effects of therapy and as the patients with proliferative DR or macular edema may be asymptomatic; its important to screen. An experienced ophthalmologist should perform the examinations in diabetec patients. Adult patients with type 1 diabetes should have initial eye examination within 5 years of diabetes diagnosis and patients with type 2 diabetes should have examination at the time of diagnosis.

There is no definite recomendation on the investigation of DR in prediabetic patients. In this study we examined prediabetes risk and rate of DR.

\section{METHODS:}

Among the patients admitted to the outpatient clinic with any complaint, $75 \mathrm{~g}$ oral glucose tolerance test (OGTT) was performed in 35 male and 65 female patients. Oral glucose tolerance test was applied to patients with a fasting blood glucose of $100-126 \mathrm{mg} / \mathrm{dl}$, obese, unexplained retinopathy, neuropathy and coronary disease or a history of gestational diabetes. These patients who underwent OGTT test were examined concurrently eye examination by an experienced ophthalmologist.

A complete opthalmic examination including visual acuity, intraocular pressure measurement (Goldmann applanation), anterior segment and fundus examinations, central foveal thickness (CFT), mean foveal thickness (MFT) measurements were performed for each patients. CFT and MFT measurements were done by Spectral domain optical coherence tomography (SD-OCT) (Optovue Inc., Fremont, CA, USA). The SD-OCT measurements (Optovue Inc., Fremont, CA, USA) were performed in a dim room after mydriasis with tropicamide $0.5 \%$ drops All SD-OCT assessments were performed by one of the authors. Mean outcome measures were central foveal thickness (CFT), mean foveal thickness (MFT) calculated automatically by SD-OCT.

\section{CONCLUSION:}

Eye examination revealed no retinopathy in any patient. DR is the specific microvascular complication of DM and affects 1 in 3 persons with DM. In many countries, DR is the most frequent cause of preventable blindness in working-aged adults. Screening for DR is an important aspect of DM management worldwide. A screening exam could include a complete ophthalmic examination with refracted visual acuity and state-of-the retinal imaging.

Providing optimal glycemic control reduces diabetic retinopathy or slows the development of retinopathy. Therefore, even if retinopathy is not detected in prediabetic patients, follow-up of these patients and reduction of diabetes risk are very important in preventing visual loss.

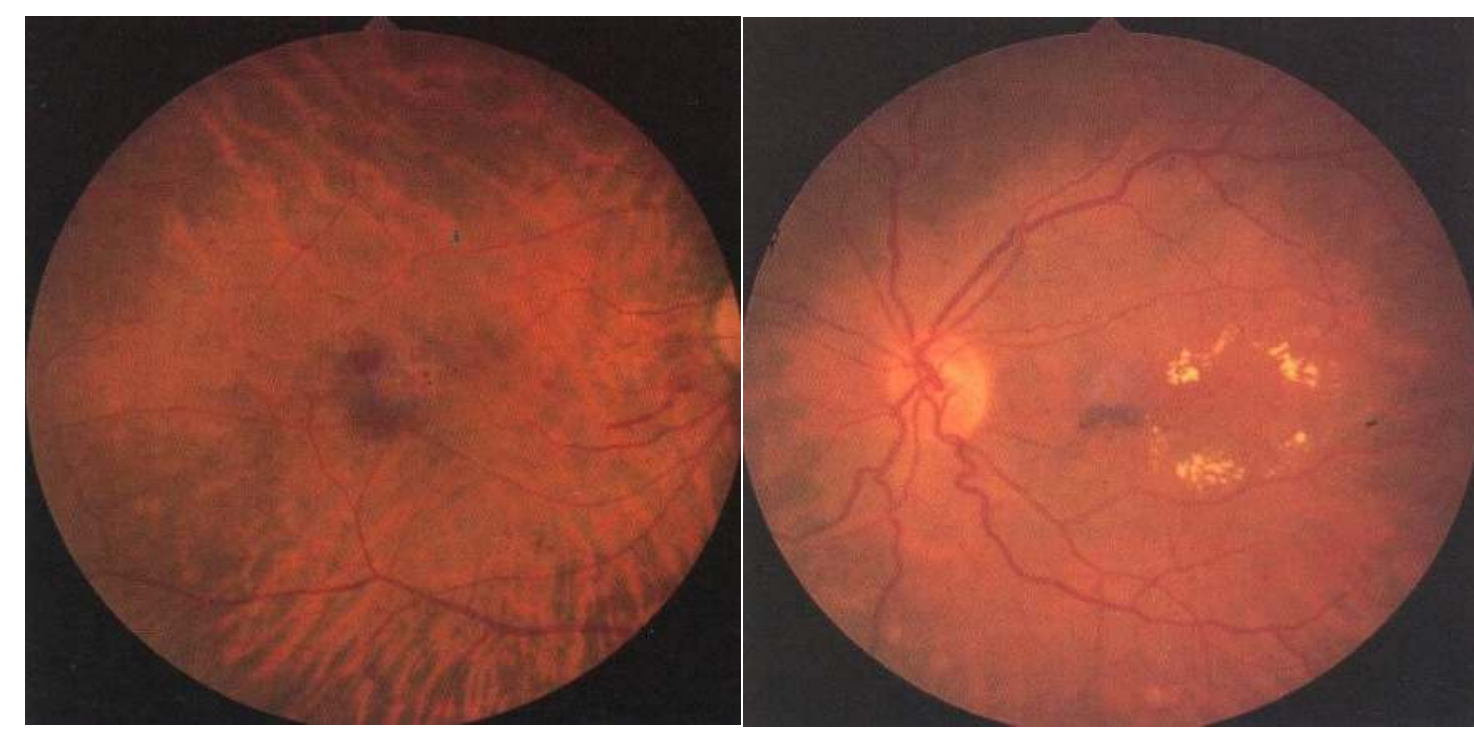

Figure 1: Early nonproliferative diabetic retinopathy, NPDR, retinal hemorrhages (left), Moderate NPDR, retinal hemorrhage, macular edema, and macula edema (right).

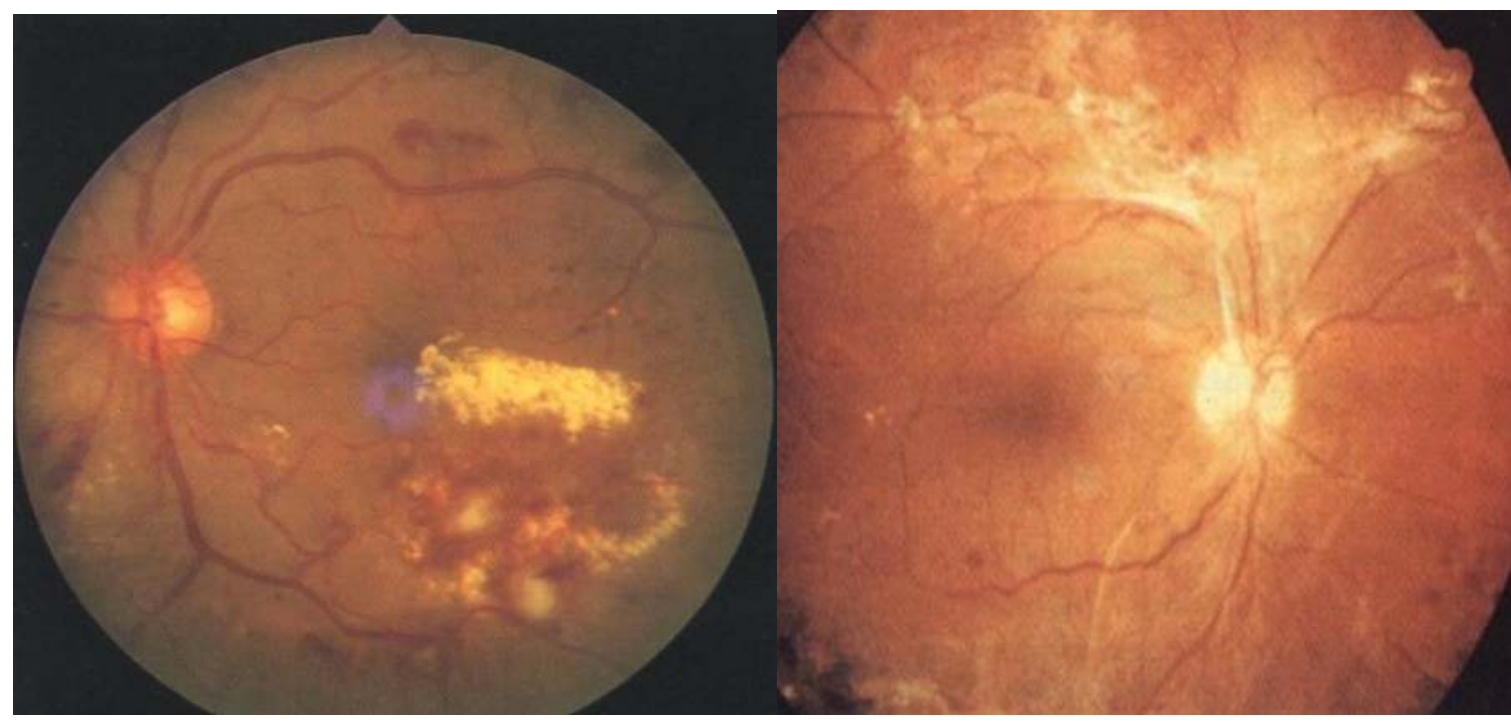

Figure 2: Proliferative diabetic retinopathy. (PDR ) Retinal neovascularization, stiff exudates, preretinal hemorrhage (left), Advanced PDR: fibrovascular bands causing retinal drawbacks and retinal hemorrhages (right) 